1 abdominal and 1 nasopharyngeal). All 7 dogs were male, with a mean age of 6 years and were of assorted breeds with variation in body surface area (BSA) from 0.63 to $1.55 \mathrm{~m}^{2}$. The overall response rate was 100\% (100\% complete response). The median PFI was 93 days (range $43-730$ days) and median overall survival time was 146 days (range 51 to $730+$ days). Four dogs required changes in their protocol due to adverse effects (1 due to neutropenia associated with vincristine, 2 due to elevations in liver enzymes and 1 due to owner perceived reduction in dog's quality of life). The median time taken to protocol change was 76 days (range 27-139 days). One dog had to be hospitalised and 3 dogs required treatment delays due to neutropenia. $\mathrm{PFI}$ is similar to that previously reported with lomustine based chemotherapy protocols but these results show an improved complete response rate when compared to previous studies.

\section{Risk of development of keratoconjunctivitis sicca in dogs with sinonasal and maxillary tumours treated with radiotherapy}

\section{Irina Gramer, Laura Blackwood}

University of Liverpool, Small Animal Teaching Hospital, Neston, Wirral, UK

\section{INTRODUCTION}

Radiotherapy $(\mathrm{RT})$ is commonly used to treat canine sinonasal and maxillary tumours. However, these tumours are adjacent to important, normal organs at risk (OAR) including ocular structures, the lacrimal gland, and forebrain. KCS is a recognized complication of sinonasal/maxillary irradiation, but its frequency is unknown. This retrospective study aimed to determine the frequency of KCS, period of risk and possible risk factors.

\section{MATERIAL AND METHODS}

A total of 59 dogs treated with fractionated RT for sinonasal and maxillary tumours were retrospectively evaluated (2010 to 2014). In all patients RT was performed using a computer generated treatment plan at 6MV or 10MV. KCS was defined as reduced tear production ( $<15 \mathrm{~mm}$ ) on Schirmer tear test (STT). Reduced tear production was categorized into two groups: 1) mild-moderate KCS (5-15 mm) and 2) severe KCS
$(<5 \mathrm{~mm})$. The degree of KCS was correlated with the dose to the adjacent eye, as the lacrimal gland is not routinely contoured as an OAR. All dogs received artificial tears once an abnormal STT was documented.

\section{RESULTS}

Among the 59 dogs recruited, 23 different breeds were represented with a median age of nine years (3-14 years). There were fourteen different tumour types. Patients had a median follow-up time of 204 days (40-836 days). The dose to the adjacent eye ranged from 4-49Gy (mean 29.7Gy). KCS developed in $27.9 \%$ of patients with severe KCS in $15 \%$. Mild to moderate KCS was apparent in five (right eye) and eight (left) cases. Severe KCS developed in seven (right) and eight cases (left). In five dogs both eyes developed KCS. Dogs in which the ipsilateral eye received an RT dose over 35Gy were more likely to develop $\operatorname{KCS}(p=0.002)$. The median duration from RT start to KCS detection was 82 days (22283 days). In this study population no other risk factors, besides RT dose, were identified.

\section{CONCLUSION}

Using the dose to the adjacent eye as an indicator, this study identified a cut-off RT dose of 35Gy after which dogs were significantly more likely to develop KCS. This occurred as early as 22 days and as late as 283 days, warranting regular monitoring to assure adequate treatment to prevent secondary corneal ulceration. Poor performance of routine
abdominal ultrasound in the detection of visceral metastasis in a uniform population of dogs with high-risk mast cell tumours

\section{Evi Pecceu, Juan Carlos Serra, Chiara Piccinelli, Ian Handel, Elspeth Milne, Jessica Lawrence}

Edinburgh University, Royal (Dick) School of Veterinary Studies, Edinburgh, UK

Conflicting evidence exists regarding the importance of routine abdominal ultrasound (AUS) with hepatic and splenic aspiration cytology during staging of canine mast cell tumours (MCTs) but it is considered standard and vital in dogs at high risk for metastasis. Our primary hypothesis was that despite selecting for dogs with high-risk MCT, AUS is poorly predictive of visceral metastasis. Our secondary hypothesis was that routine cytology in dogs with high-risk MCT reveals metastasis in fewer than 20\% of high-risk cases. The objective of this study was to correlate ultrasonographic changes and cytologic findings during initial staging in dogs with strictly defined high-risk MCTs.

Dogs were included if they were diagnosed with a high-risk MCT between March 2013 and March 2015 and if staging included regional lymph node (LN) assessment, AUS, and aspiration cytology of the liver and spleen. Inclusion criteria for dogs with high-risk MCTs were clearly defined. Standardised cytological criteria were used to define visceral MCT metastasis.

Of 42 dogs included, 2 (5\%) had liver abnormalities on AUS whereas 15 (36\%) had splenic abnormalities. In 4 dogs, 


\section{Oral presentations}

hepatic location prohibited aspiration. Five dogs had visceral metastasis leading to an overall rate of visceral metastasis of $12 \%$. Two dogs (5\%) had liver and spleen metastasis; 2 dogs (5\%) had liver metastasis and 1 dog (2\%) had splenic metastasis. Overall sensitivity of AUS for detecting liver and splenic metastasis was 25\% and 33\%, respectively; specificity for detecting liver and splenic metastasis was $100 \%$ and $64 \%$, respectively. Positive predictive values were less than $20 \%$. Negative predictive values for detection of liver and splenic metastasis were 97\% and 93\%, respectively. Three of the 5 dogs (60\%) with visceral infiltration had regional $L N$ metastasis. When the 20 dogs with regional lymph node metastasis were considered separately, three dogs (15\%) were positive for visceral metastasis.

When strict criteria were applied to select for dogs considered at high risk for visceral metastasis, routine AUS with hepatic and splenic cytology carried low utility, particularly with normal appearance on ultrasound. The visceral metastatic rate was only $12 \%$. Selecting for dogs with regional LN metastasis did not increase the utility of routine AUS with liver and splenic aspiration. Results suggest that a paradigm change for staging and monitoring dogs with high-risk MCT may be warranted.

\section{Outcomes of multimodality treatment for canine and feline salivary gland carcinomas.}

\section{Irina Gramer, Laura Blackwood}

University of Liverpool, Small Animal Teaching Hospital, Neston, Wirral, UK

\section{INTRODUCTION}

Salivary gland carcinomas (SGC) are rare in cats and dogs. Multimodality treatment approaches including surgical excision and radiotherapy (RT) have been recommended though prognosis is poorly defined. The benefit of including chemotherapy is unknown. The present study aims at describing RT as adjuvant treatment following surgical excision of canine and feline SGCs.

\section{MATERIAL AND METHODS}

Five dogs and two cats treated with RT for histologically confirmed submandibular $(n=2)$ and parotid $(n=5)$ SGCs were retrospectively evaluated (2008 to 2014). Four cases (three dogs, one cat) received 12 fractions of $4 \mathrm{~Gy}$ on a Monday-Wednesday-Friday basis and three cases received 8-9Gy on a once weekly basis. In six cases treatment was manually planned and in one computer planned (Pinnacle). The ipsilateral submandibular lymph node was included in the radiation field in all cases. $\mathrm{RT}$ side effects were graded using the VRTOG scoring system. Median survival times
(MSTs) were calculated from the time of first presentation until death or last follow-up.

\section{RESULTS}

All tumours were adenocarcinomas with a median mitotic rate of seven (range 1-26) per ten high power fields. Metastasis was present in two dogs at the time of diagnosis. SGCs were detected about 4.8 weeks (range of 1-12 weeks) prior to referral. Median tumour size was $5.0 \mathrm{~cm}$ (range $4.5-5.5 \mathrm{~cm}$ ) for dogs and $1.6 \mathrm{~cm}$ (range $1.5-1.8 \mathrm{~cm}$ ) for cats. All patients underwent surgical excision, then radiotherapy for microscopic disease. RT associated side effects occurred in all cases but were generally mild (acute side effects grade 1, $n=5$; grade $2, n=1$; late side effects grade $1, n=2)$. Three of the patients were euthanized due to progressive disease, and four had MST estimated on the basis of last follow-up. ST for dogs was 425 days (range 107-794 days) and 260 and 767 days for the two cats. Adjuvant carboplatin was prescribed in two dogs and one cat (two dogs with metastasis, one cat with high mitotic index). Progressive disease developed in both dogs and alternative chemotherapy was subsequently given. A decreased survival time was generally observed in animals with metastasis at the time of diagnosis and high mitotix index ( $>4$ per 10 high power fields).

\section{CONCLUSION}

Multimodality treatment of SGCs with surgery and RT with or without chemotherapy can provide reasonably long survival times.
Use of (90) strontium plesiotherapy for local control in dogs and cats with nonsquamous cell carcinoma malignancies: 10 cases

\section{Samantha Vazquez-sanmartin, Sara Verganti, Sue Murphy}

Animal Health Trust, Newmarket Suffolk, UK
(90) Strontium plesiotherapy (Sr90) has been used in Veterinary Medicine as primary local treatment for superficial squamous cell carcinomas of the nasal planum in cats and as adjuvant treatment for limbal melanomas in dogs.

The aim of this small case series is to describe the use of Sr90 as local treatment in cats and dogs with different types of malignancies (non-squamous cell carcinoma neoplasias). The data retrieved from the clinical records of a referral centre between January 2005 and October 2015 revealed a total of 10 cases, 4 dogs and 6 cats.

Six cases ( 3 dogs and 3 cats) had eye lid tumours: two melanomas, two soft tissue sarcomas, one mast cell tumour and one progressive dendritic histiocytosis. Three cases had tumours located in the paw: one feline sarcoid, one mast cell tumour (dog) and one soft tissue sarcoma (cat). 